\title{
PROBING CLINICAL RELEVANCE: ESTABLISHING THE EFFICACY OF C. NOVYI AGAINST A PANEL OF 2D CULTURED PANCREATIC CANCER CELLS
}

\author{
$\underline{\text { K.M. Dailey }}^{1}$, R.I. Jacobson ${ }^{2}$, J. Kim ${ }^{1,2}$, S. Mallik ${ }^{1}$, and A.E. Brooks ${ }^{1,3}$ \\ ${ }^{1}$ Cell and Molecular Biology Program, North Dakota State University, Fargo, ND \\ ${ }^{2}$ Department of Biological Sciences, North Dakota State University, Fargo, ND \\ ${ }^{3}$ Office of Research and Scholarly Activity, Rocky Vista University, Ivins, UT
}

Corresponding Author: Emily Dosmar

Email: dosmare@rose-hulman.edu

https://doi.org/10.34107/BiomedSciInstrum.57.0492

\begin{abstract}
Pancreatic cancer presents a unique challenge for the development of effective oncotherapies. The tumor microenvironment (TME) of this type of tumor typically contains a dense desmoplastic barrier composed of aberrant extracellular matrix proteins, as well as an acidic, hypoxic and necrotic core. Additionally, the immune system surrounding this type of tumor has often been suppressed by the TME. Hence, choosing the correct model of the tumor microenvironment within which to test a potential anti-cancer therapy is a critical experimental design decision. While the typical solid tumor contains a complex microenvironment including both phenotypic and genotypic heterogeneity, the methods used to model this disease state often do not reflect this complexity. This simplistic approach may have contributed to stagnant five-year survival rates experienced over the past four decades. Oncolytic bacteria, a class of bacteria with the innate ability to seek and destroy solid tumors has been revived from historical anecdotes in an attempt to overcome these challenges. Regardless of the promise of oncolytic bacteria, accurate assessment of their potential requires choosing the proper tumor model. This study explores the impact of cancer cell lines co-cultured with Wild-Type C. novyi to establish the efficacy of this oncolytic bacteria in a monolayer culture.
\end{abstract}

Keywords: Oncolytic bacteria, Clostridium novyi, C. novyi, Monolayer cell culture, Clinical translation, Pre-clinical modeling, Cancer, Oncotherapeutics, Pancreatic cancer, Tumor microenvironment

\section{INTRODUCTION}

Only around five percent of patients diagnosed with a pancreatic tumor survive the next five years - a staggering statistic that has not changed in four decades [1], [2]. This stagnant statistic stands in stark contrast to the significantly improved outlooks for most other major cancer types [1], [2]. In large part, the difficulty in treating this particular type of solid tumor is due to several challenging aspects of the tumor microenvironment (TME). While these characteristics are commonly found in other solid tumors, they are considered almost ubiquitous for pancreatic tumors, presenting unique and inescapable challenges for developing effective oncotherapies. The TME of a pancreatic tumor is typically characterized by a dense abnormal extracellular matrix forming a desmoplastic barrier [3], [4]. This dense structure not only limits the number of patients who are candidates for surgical tumor removal, but also inhibits the diffusion of traditional chemotherapeutics into the center of the tumor [3]. Furthermore, the centers of solid tumors are very distant from any blood vessels, and thus distant from the delivery of oxygen or nutrients as well as the removal of toxic metabolic byproducts. This distance from blood vessels ultimately results in a highly acidic, necrotic tumor core that continues to confound current oncotherapeutics [5], and predisposes 